\title{
A novel biosynthetic scaffold mesh reinforcement affords the lowest hernia recurrence in the highest-risk patients
}

Mitchell J. Parker, 1

Rachel C. Kim, 1

Martin Barrio, 1

Juan Socas, 1

Lawrence R. Reed, 1

Attila Nakeeb, 1

Michael G. House, 1

Eugene P. Ceppa, 1凶

Email eceppa@iupui.edu

1 Department of Surgery, Indiana University School of Medicine, 545

This is the author's manuscript of the article published in final edited form as:

Parker, M. J., Kim, R. C., Barrio, M., Socas, J., Reed, L. R., Nakeeb, A., House, M. G., \& Ceppa, E. P. (2021). A novel biosynthetic scaffold mesh reinforcement affords the lowest hernia recurrence in the highest-risk patients. Surgical Endoscopy, 35(9), 5173-5178. https://doi.org/10.1007/s00464-020-08009-1 
Barnhill Dr., EH541, Indianapolis, IN, 46202 USA

Received: 26 May 2020 / Accepted: 16 September 2020

\section{Abstract}

\section{Introduction}

Patients with higher postoperative infection risk undergoing ventral hernia repair (VHR) have limited options for mesh use. Biosynthetic mesh is intended to utilize the durability of synthetic mesh combined with the biocompatibility of biologic mesh. We sought to assess the outcomes of a novel biosynthetic scaffold mesh for VHR in higher risk patients over a 12month postoperative period.

\section{Methods}

Two cohorts of 50 consecutive patients who underwent VHR with TELA Bio OviTex biosynthetic or synthetic mesh were retrospectively compared. Endpoints included surgical site occurrence (SSO), readmission rate, and hernia recurrence following VHR at 12 months postoperatively.

\section{Results}

OviTex mesh placement was associated with higher risk Ventral Hernia Working Group (VHWG) distribution and more contaminated CDC wound class distribution compared to synthetic mesh placement (VHWG grade 3: $68 \%$ vs. $6 \%, p<0.001 ;$ CDC class $>$ I: $70 \%$ vs. $6 \%, p<0.001)$. Additionally, concomitant procedures were performed more often with OviTex mesh placement than synthetic mesh placement $(70 \%$ vs $10 \%$, p < 0.001). The OviTex mesh performed comparably to synthetic mesh in terms of incidences of SSO (36\% vs 22\%, $p=0.19)$, readmission rates $(24 \%$ vs 
$14 \%, p=0.31$ ), and hernia recurrence ( $6 \%$ vs $12 \%, p=0.74)$. On further evaluation, patients who developed SSO with OviTex mesh $(n=18)$ had a $17 \%$ hernia recurrence whereas those with synthetic mesh $(n=11)$ had an associated 55\% hernia recurrence $(p=0.048)$.

\section{Conclusions}

The OviTex biosynthetic mesh was used in higher risk patients and performed similarly to synthetic mesh in regards to rate of SSO, readmissions, and hernia recurrence. Furthermore, patients who developed SSO with Ovitex mesh were significantly less likely to have hernia recurrence than those with synthetic mesh. Overall, the data suggest that biosynthetic mesh is a more desirable option for definitive hernia repair in higher risk patients.

\section{Keywords}

Ventral hernia repair

Biosynthetic hybrid mesh

Contaminated ventral hernia repair

Synthetic mesh

TELA bio OviTex

Abbreviations

VHR Ventral hernia repair

VHWG Ventral Hernia Working Group

CDC Centers for Disease Control and Prevention

BMI Body mass index

SSO Surgical site occurrence

LOS Length of stay

CPT Current procedural terminology

This paper is a combination of two abstracts that have been presented as posters at both the 2018 Western Surgical Association Meeting in San Jose del 
Cabo, Mexico and the 2019 Americas Hernia Society Meeting in Las Vegas, NV, USA.

Several variables must be considered when selecting mesh to augment ventral hernia repair (VHR). Understanding patient comorbidities, potential for infection, and how the material will incorporate at a cellular level will increase success and avoid surgical morbidity. Universal criteria for material selection include but are not limited to non-carcinogenicity, chemical inertness, resistance to mechanical stress, sterility, unresponsiveness to body and tissue fluids, limitation of foreign-body reactions, modifiability in size, and non-allergenicity [1]. In addition to material considerations, sound clinical reasoning must be applied to aspects such as patient comorbidities, social history, and infection risk when contemplating mesh use in hernia repair. In order to better risk-stratify patients in predicting surgical site occurrences (SSO), the Ventral Hernia Working Group (VHWG) created a classification system for incisional hernias [2]. More recently, Kanters et al. devised a modified VHWG classification system to improve the accuracy of predicting SSO after open hernia repair [3]. Increased SSO risk is associated with higher hernia grades in the classification system.

With evolving technology and techniques, the selection of mesh seems almost limitless. Traditionally, the selection had been between synthetic or biologic materials, each containing respective strengths and weaknesses. As an overview, synthetic materials such as polypropylene, polyester, or expanded polytetrafluoroethylene (ePTFE) generally maintain higher tensile strength yet carry a higher infection risk with regards to foreign-body reactions such as chronic inflammation, fibrosis, abdominal wall stiffness, and fistulas $[4,5,6]$. Biologic mesh derived from human, bovine, or porcine tissue addresses foreign-body infection risks yet are scrutinized for high cost and lack of strength leading to higher hernia recurrence as the graft is resorbed over time $[4,5,6]$. With the strengths and weaknesses of these materials at the extremes of a continuum, patients with higher risk of infection assume the risk yet may not achieve the benefit of each category of mesh. Therefore, new approaches and material amalgamation are required for improved outcomes. 
Biosynthetic, and/or hybrid, mesh are comprised of long-term absorbable synthetic materials or are meshes that incorporate both biologic and synthetic components, which have been shown to be effective in clean contaminated and contaminated wounds $[6,7,8]$. The principal goal of hybrid mesh is to establish a scaffold for tissue ingrowth in addition to maintaining integrity with a permanent synthetic support. This category of mesh is designed to stimulate fibroblast migration in addition to cell signaling cascades, leading to neovascularization and deposition of collagen $[6,7,8]$. One such biosynthetic option is the TELA Bio OviTex Reinforced Bioscaffolds. The reinforced bioscaffold consists of layers of biologic tissue consisting of ovine rumen that is interwoven with a monofilament polypropylene. The biologic component allows for reduced foreign body responses, decreased inflammation, and enhancement of host tissue remodeling while the interwoven synthetic filament allows for increased strength and lower hernia recurrence. There is no literature describing outcomes of high-risk ventral hernia repair utilizing OviTex biosynthetic mesh, thus we sought to assess the surgical outcomes in this patient population. The aims of this study were to assess the SSO, hernia recurrence, and hospital readmission rates with respect to higher risk patients receiving OviTex biosynthetic mesh.

\section{Methods}

\section{Patient population}

All patients were identified using CPT procedural codes corresponding to open VHR using either TELA Bio OviTex biosynthetic or synthetic meshes. All synthetic meshes used were microporous, low-weight, composite-type polypropylene-based mesh with an absorbable barrier. Patients underwent surgery at either Indiana University Health Methodist or University Hospitals during the 2017 calendar year. Cases from multiple surgeons of multiple specialties were included, including one trauma surgeon, one plastic surgeon, and three gastrointestinal surgeons. Data were collected from retrospective chart review and readmissions, clinic visits, and complications were documented in a 12-month post-operative period. Patients were stratified 
using the modified VHWG grading classification (Fig. 1) and CDC wound classifications I-IV (Fig. 2). Exclusion criteria included patients less than 18 years of age, patients receiving biologic or other forms of biosynthetic mesh other than OviTex, and patients who were lost to follow-up. IRB approval was obtained for this study.

\section{Fig. 1}

Modified VHWG classification system as defined by Kanters et al. [3]

\begin{tabular}{l|l|l}
\hline \multicolumn{3}{c}{ Modified Ventral Hernia Working Group Grading System } \\
\hline Grade 1 & Grade 2 & Grade 3 \\
\hline Low Risk & Co-morbidities & Contaminated \\
Low risk of complications & Smoking & Clean contamir \\
No history of wound infection & Obesity & Contaminated \\
& Diabetes & Active infection \\
& Immunosuppression & \\
& Previous wound infection & \\
\hline
\end{tabular}

\section{Fig. 2}

Surgical wound classification grades as defined by the CDC 


\begin{tabular}{|c|c|}
\hline $\begin{array}{l}\text { Class I } \\
\text { Clean }\end{array}$ & $\begin{array}{l}\text { An uninfected operative wound in which no inflammation is encot } \\
\text { and the respiratory, alimentary, genital, or uninfected urinary trac } \\
\text { entered. In addition, clean wounds are primarily closed and, if nec } \\
\text { drained with closed drainage. Operative incisional wounds that fol } \\
\text { nonpenetrating (blunt) trauma should be included in this category } \\
\text { meet the criteria. }\end{array}$ \\
\hline $\begin{array}{l}\text { Class II } \\
\text { Clean-contaminated }\end{array}$ & $\begin{array}{l}\text { An operative wound in which the respiratory, alimentary, genital, } \\
\text { urinary tracts are entered under controlled conditions and withou } \\
\text { unusual contamination. Specifically, operations involving the bilia } \\
\text { appendix, vagina, and oropharynx are included in this category, pr } \\
\text { no evidence of infection or major break in technique is encounter }\end{array}$ \\
\hline $\begin{array}{l}\text { Class III } \\
\text { Contaminated }\end{array}$ & $\begin{array}{l}\text { Open, fresh, accidental wounds. In addition, operations with majo } \\
\text { in sterile technique (e.g., open cardiac massage) or gross spillage f } \\
\text { gastrointestinal tract, and incisions in which acute, nenpurulent } \\
\text { inflammation is encountered are included in this category. }\end{array}$ \\
\hline $\begin{array}{l}\text { Class IV } \\
\text { Dirty-infected }\end{array}$ & $\begin{array}{l}\text { Old traumatic wounds with retained devitalized tissue and those } t \\
\text { involve existing clinical infection or perforated viscera. This definit } \\
\text { suggests that the organisms causing postoperative infection were } \\
\text { in the operative field before the operation. }\end{array}$ \\
\hline
\end{tabular}

\section{Outcomes}

Individual data on each patient were collected including age, gender, BMI, ethnicity, comorbidities, tobacco use (current or past), hospital LOS (index and total), past surgical history, hernia size, repair method, and concomitant surgeries. Endpoints of interest included SSO, readmission rate, and hernia recurrence at more than 12 months post-operatively. An SSO was defined as any defect within the midline incision including abscess, seroma, dehiscence, hematoma, or cellulitis. 


\section{Statistical analyses}

Statistical analysis was performed using SPSS software, version 24 (IBM corporation; Armonk, NY) and R, version 3.5.2 (R Foundation for Statistical Computing) to identify significant differences between two 50-patient cohorts receiving either OviTex biosynthetic or poly-propelene synthetic meshes. Categorical data were displayed as percentages and continuous data presented as averages with standard deviations. Categorical data were analyzed using a $2 \times 2$ contingency table with a two-tailed Fisher's Exact Test (FET). Continuous data were analyzed with unpaired $t$-tests. Statistical significance was defined as $p<0.05$.

\section{Results}

\section{Biosynthetic vs. synthetic outcomes}

Two cohorts of 50 consecutive patients who underwent VHR with OviTex biosynthetic or synthetic mesh were compared (Table 1). No biosynthetic mesh patients were lost to follow-up, meanwhile five synthetic mesh patients did not have 12 months follow-up and were excluded. Of the patients receiving OviTex biosynthetic mesh, the majority of patients were female (58\%), mean age of $55 \pm 14$ years, and mean body mass index (BMI) of $34 \pm$ $6 \mathrm{~kg} / \mathrm{m}^{2}$. Mean defect size was $124 \pm 63 \mathrm{~cm}^{2}$, with $68 \%$ requiring component separation. Primary fascial closure was achieved in $92 \%$ of cases and location of mesh included underlay (68\%), sublay (20\%), and onlay (12\%).

Concomitant procedures were performed in $70 \%$ of patients. VHWG distribution included grade $2(32 \%)$ and grade $3(68 \%)$. Wound class distribution included CDC class I (30\%), II (44\%), III (10\%), and IV (16\%). SSO were seen in $36 \%$ of patients, which included seroma $(n=5)$, abscess/deep SSI $(n=8)$, and wound drainage/dehiscence $(n=5)$. Average LOS distribution in days included ICU (1) and total (11). Rate of readmission was $24 \%$ and hernia recurrence rate was $6 \%(n=3)$. Open VHR with synthetic mesh group had a similar mean age and BMI. Mesh location included underlay (62\%), sublay (34\%), and onlay (4\%). Wound class consisted CDC class I (94\%), II (4\%), and III (2\%); VHWG included grade 2 (94\%) and grade 3 (6\%). Postoperative occurrences included 22\% SSO, 14\% 
readmission rate, and $12 \%(n=6)$ hernia recurrence, which were comparable to that of the OviTex mesh cohort. OviTex mesh was associated with a significantly higher VHWG distribution, higher CDC wound classification, and longer length of stay compared to synthetic mesh. An attempt was made to match preoperative and operative variables of contemporary patients but was unsuccessful due to the lack of patients with synthetic mesh and VHWG grade 3 , thus we reported consecutive patient data. 
Table 1

Comparison of patient data receiving OviTex TELA Bio mesh vs. synthetic mesh

\begin{tabular}{|c|c|c|c|}
\hline & OviTex TELA Bio & Synthetic mesh & $p$ value \\
\hline$N$ & 50 & 50 & \\
\hline Gender & $\begin{array}{l}21 \mathrm{M}(42 \%) \\
29 \mathrm{~F}(58 \%)\end{array}$ & $\begin{array}{l}27 \mathrm{M}(54 \%) \\
23 \mathrm{~F}(46 \%)\end{array}$ & 0.32 \\
\hline Age (years) & $55 \pm 14$ & $52 \pm 12$ & 0.25 \\
\hline BMI $\left(\mathrm{kg} / \mathrm{m}^{2}\right)$ & $34 \pm 6$ & $33 \pm 7$ & 0.45 \\
\hline VHWG grade & $\begin{array}{l}2(32 \%) \\
3(68 \%)\end{array}$ & $\begin{array}{l}2(94 \%) \\
3(6 \%)\end{array}$ & $\begin{array}{l}<0.001 * \\
<0.001 *\end{array}$ \\
\hline CDC wound class & $\begin{array}{l}\text { I }(30 \%) \\
\text { II }(44 \%) \\
\text { III }(10 \%) \\
\text { IV }(16 \%)\end{array}$ & $\begin{array}{l}\text { I }(94 \%) \\
\text { II }(4 \%) \\
\text { III }(2 \%) \\
\text { IV }(0 \%)\end{array}$ & $\begin{array}{l}<0.001^{*} \\
<0.001^{*} \\
0.20 \\
0.006^{*}\end{array}$ \\
\hline Repair method & Open & Open & \\
\hline Mesh location & $\begin{array}{l}\text { Underlay }(68 \%) \\
\text { Sublay }(20 \%) \\
\text { Onlay }(12 \%)\end{array}$ & $\begin{array}{l}\text { Underlay }(62 \%) \\
\text { Sublay }(34 \%) \\
\text { Onlay }(4 \%)\end{array}$ & $\begin{array}{l}0.68 \\
0.18 \\
0.27\end{array}$ \\
\hline Concomitant surgeries & $70 \%$ & $10 \%$ & $<0.001 *$ \\
\hline Avg. hospital LOS (days) & 11 & 2 & $<0.001^{*}$ \\
\hline SSO & $36 \%$ & $22 \%$ & 0.19 \\
\hline Readmission rate & $24 \%$ & $14 \%$ & 0.31 \\
\hline Hernia recurrence & $6 \%$ & $12 \%$ & 0.74 \\
\hline
\end{tabular}

\section{Surgical site occurrences (SSO) outcomes}

Data from both cohorts with respect to patients who developed SSO are found in Table 2 . The OviTex cohort with SSO $(n=18 ; 36 \%)$ consisted mostly of VHWG grade 3 (61\%) and CDC wound class III (61\%). Concomitant 
procedures were performed in $67 \%$ of these patients, and the average hospital LOS was 11 days. On the other hand, the synthetic cohort with SSO $(n=11$; $22 \%)$ consisted of mostly VHWG grade $2(91 \%)$ and CDC wound class I (91\%). Only 9\% underwent concomitant procedures, and the average LOS was 3 days. Most notably, only 17\% of patients with OviTex mesh who developed SSO also developed hernia recurrence, in contrast to $55 \%$ of patients with synthetic mesh and SSO $(p=0.048)$.

\section{Table 2}

Comparison of patient data receiving OviTex TELA Bio mesh vs. synthetic mesh who sustained a surgical site occurrence (SSO)

$\left.\begin{array}{|l|l|l|l|}\hline & \text { OviTex SSO } & \text { Synthetic SSO } & p \text { value } \\ \hline N & 18(36 \%) & 11(22 \%) & 0.187 \\ \hline \text { Gender } & \begin{array}{l}\text { M (11\%) } \\ 16 \mathrm{~F}(89 \%)\end{array} & \begin{array}{l}7 \mathrm{M}(64 \%) \\ 4 \mathrm{~F}(36 \%)\end{array} & 0.010^{*} \\ \hline \text { Age (years) } & 49 \pm 14 & 47 \pm 11 & 0.648 \\ \hline \text { BMI }\left(\mathrm{kg} / \mathrm{m}^{2}\right) & 35 \pm 7 & 34 \pm 9 & 0.893 \\ \hline \text { VHWG grade } & \begin{array}{l}2(39 \%) \\ 3(61 \%)\end{array} & \begin{array}{l}2(91 \%) \\ 3(9 \%)\end{array} & 0.008^{*} \\ \hline \text { CDC wound class }>\text { I } & 61 \% & 9 \% & 0.008^{*} \\ \hline \text { Tobacco use } & 78 \% & 73 \% & 1.0 \\ \hline \text { Mesh location } & \begin{array}{l}\text { Underlay }(56 \%) \\ \text { Sublay }(33 \%) \\ \text { Onlay }(11 \%)\end{array} & \begin{array}{l}\text { Underlay }(73 \%) \\ \text { Sublay }(27 \%)\end{array} & 0.449 \\ \hline \text { Onlay }(0 \%)\end{array}\right)$




\section{Discussion}

Ventral hernia repair remains one of the most commonly performed surgical procedures, with over 350,000 cases performed annually in the United States. The surgery unfortunately carries high rates of recurrence ranging from 10 to $32 \%$. These recurrences can be extremely costly to both the patient and the hospital, and it has been estimated that reducing recurrence rates by even $1 \%$ could save the nation millions of dollars in healthcare costs $[8,9,10]$. Factors that can play a role in hernia recurrence include patient characteristics such as obesity, tobacco consumption, and wound contamination, as well as operative factors such as open vs. laparoscopic entry and mesh selection. As more surgical options and mesh materials become available, the best approach is often unclear and continuously evolving. This study focused on a novel biosynthetic hybrid mesh in highly comorbid patients in a single-institution retrospective review. This study in particular is one of the first to focus on the TELA Bio OviTex biosynthetic scaffold mesh in patients classified as VHWG grades 2 and 3 as compared to patients receiving synthetic mesh for VHR.

The results of the study demonstrated that surgeons at our institution were more likely to select the OviTex biosynthetic mesh over a pure synthetic mesh in higher risk patients and cases, such as those with a higher degree of wound contamination or those performed with concomitant surgeries. Despite that, the data showed no significant differences in rates of SSO, readmissions, or hernia recurrence between the two groups. Average hospital length of stay was significantly increased in the OviTex cohort. However, this may be attributed to increased usage of the mesh in higher risk patients and more involved surgeries. Most importantly, on further analysis of patients who developed SSO, the OviTex mesh demonstrated statistically significant lower rates of hernia recurrence than synthetic mesh.

Investigating safe and effective approaches to hernia repairs in high risk patients is critical for their management. Repair of a hernia is often an elective or semi-elective procedure, and in such circumstances, surgeons will attempt to optimize the patient's medical and surgical risks. However, hernia repair is also commonly an urgent or emergent procedure when incarceration, 
bowel ischemia, or acidosis/shock are involved. Pre-existing active infection or wound contamination is considered a contraindication to synthetic mesh placement, due to increased infection risk, yet primary tissue repair alone of hernia defects has been repeatedly demonstrated to be associated with higher risks of recurrence $[11,12]$. Biologic meshes, on the other hand, are significantly more costly and carry a higher risk of hernia recurrence compared to synthetic mesh $[4,5,6]$. The results of our study are promising as they suggest that the Ovitex biosynthetic mesh could be a better option for definitive hernia repair in the highest risk cases, such as those with higher preoperative risk or increased degree of contamination.

The literature supporting the use of OviTex biosynthetic scaffold mesh in VHR is extremely limited. Lake et al. studied hybrid hernia meshes, including the OviTex resorbable bioscaffold, in rabbit bacterial inoculated models and demonstrated microbial colonization of the OviTex mesh at 7 days postinoculation [13]. To date there have been no studies investigating the clinical significance of these findings with regards to outcomes such as mesh infection in humans. A case series by Ferzoco that focused on inguinal hernia repair with the TELA Bio OviTex mesh demonstrated no reported hernia recurrence in an average 12-month postoperative period. The patients reported no complications, including no SSO nor recurrence, as well as a decrease in post-operative pain [14]; these findings were not surprising considering the low rate of morbidity and recurrence for inguinal herniorraphy regardless of mesh selection. Our study corroborated the safety and effectiveness of the OviTex mesh for ventral hernia. Furthermore, the data reported here are the first to investigate surgical outcomes in high-risk patients undergoing open and often complex (requiring component separation) abdominal wall hernia repairs.

Several limitations exist for the study including its retrospective design at a single institution over a 1-year time period, which does not account for a selection bias in terms of mesh used by surgeons in a consecutive series of hernia repairs. An effort to limit the influence of this bias was made by collecting data from multiple surgeons across multiple specialties and 
hospitals, including one trauma surgeon, one plastic surgeon, and three gastrointestinal surgeons. An attempt was also made to match the VHWG grade and CDC wound classification of contemporary cases several years before the use of OviTex; the primary limitation was that other forms of biologic mesh were used instead for these same patients with VHWG grade 3 and CDC wound classification II-IV. Data collection was performed via retrospective chart review and does not exclude the possibility of a confirmation bias; follow up at 12-months was felt to be reasonable in both groups based on clinic visits and available cross-sectional imaging. Although consecutive patients were used in data collection, considerable variance existed including multiple surgeons with varying surgical approaches as well as location of the mesh placement. The study had 100 total patients ( $n=50$ for both synthetic and biosynthetic mesh use), and thus is limited by a relatively small sample size. Further prospective, multi-center trials utilizing TELA Bio OviTex mesh for open VHR are needed to support our results. One such trial has completed enrollment and hernia repair, and 24-month follow-up is currently ongoing (NCT03074474). Overall, the optimal approach and mesh selection for complicated VHR remains unclear in the literature and requires continued investigation.

\section{Conclusions}

Although numerous categories of mesh are available for ventral hernia repair, the options become limited in severely comorbid or contaminated cases. Our data suggested that TELA Bio OviTex biosynthetic mesh is a safe option in VHWG grade 2 and 3 patients in which synthetic mesh would be contraindicated with comparable rates of SSO and hernia recurrence at 12 months follow-up. OviTex biosynthetic mesh offers durable defect reinforcement with a decreased risk for hernia recurrence in comparison to synthetic mesh options in high risk patients. Further prospective multi-center trials utilizing TELA Bio OviTex mesh for open VHR are ongoing and needed to support the validity of these results.

\section{Publisher's Note}


Springer Nature remains neutral with regard to jurisdictional claims in published maps and institutional affiliations.

Compliance with ethical standards

Disclosures Mitchell J. Parker, Rachel C. Kim, Martin Barrio, Juan Socas, Lawrence R. Reed, Attila Nakeeb, Michael G. House and Eugene P. Ceppa declares that they have no conflicts of interest or financial ties to disclose.

\section{References}

1. Bryan N, Battersby C, Smart N, Hunt J (2015) A review of biocompatibility in hernia repair; considerations in vitro and in vivo for selecting the most appropriate repair material. Hernia 19:169-178

2. Breuing K, Butler CE, Ferzoco S, Franz M, Hultman CS, Kilbridge JF, Rosen M, Silverman RP, Vargo D (2010) Incisional ventral hernias: review of the literature and recommendations regarding the grading and technique of repair. Surgery 148:544-558

3. Kanters AE, Krpata DM, Blatnik JA, Novitsky YM, Rosen MJ (2012) Modified hernia grading scale to stratify surgical site occurrence after open ventral hernia repairs. J Am Coll Surg 215:787-793

4. FitzGerald JF, Kumar AS (2014) Biologic versus synthetic mesh reinforcement: what are the pros and cons? Clin Colon Rectal Surg $27: 140-148$

5. Bondre IL, Holihan JL, Askenasy EP, Greenberg JA, Keith JN, Martindale RG, Roth JS, Liang MK (2016) Suture, synthetic, or biologic in contaminated ventral hernia repair. J Surg Res 200:488-494

6. Reid CM, Jacobsen GR (2018) A current review of hybrid meshes in abdominal wall reconstruction. PlastReconstrSurg 142:92s-96s 
7. Rosen MJ, Bauer JJ, Harmaty M, Carbonell AM, Cobb WS, Matthews B, Goldblatt MI, Selzer DJ, Poulose BK, Hansson BM, Rosman C, Chao JJ, Jacobsen GR (2017) Multicenter, prospective, longitudinal study of the recurrence, surgical site infection, and quality of life after contaminated ventral hernia repair using biosynthetic absorbable mesh: the COBRA study. Ann Surg 265:205-211

8. Sahoo S, Haskins IN, Huang LC, Krpata DM, Derwin KA, Poulose BK, Rosen MJ (2017) Early wound morbidity after open ventral hernia repair with biosynthetic or polypropylene mesh. J Am Coll Surg 225:472-480.e471

9. Poulose BK, Shelton J, Phillips S, Moore D, Nealon W, Penson D, Beck W, Holzman MD (2012) Epidemiology and cost of ventral hernia repair: making the case for hernia research. Hernia 16:179-183

10. Baucom RB, Ousley J, Feurer ID, Beveridge GB, Pierce RA, Holzman MD, Sharp KW, Poulose BK (2016) Patient reported outcomes after incisional hernia repair-establishing the ventral hernia recurrence inventory. Am J Surg 212:81-88

11. Burger JW, Luijendijk RW, Hop WC, Halm JA, Verdaasdonk EG, Jeekel J (2004) Long-term follow-up of a randomized controlled trial of suture versus mesh repair of incisional hernia. Ann Surg 240:578-583 discussion 583-575

12. Luijendijk RW, Hop WC, van den Tol MP, de Lange DC, Braaksma MM, IJzermans JN, Boelhouwer RU, de Vries BC, Salu MK, Wereldsma JC, Bruijninckx CM, Jeekel J (2000) A comparison of suture repair with mesh repair for incisional hernia. N Engl J Med 343:392-398

13. Lake SP, Stoikes NFN, Badhwar A, Deeken CR (2019) Contamination of hybrid hernia meshes compared to bioresorbable Phasix ${ }^{\mathrm{TM}}$ Mesh in a rabbit subcutaneous implant inoculation model. Ann Med Surg 46:12-16 
14. Ferzoco SJ (2018) Early experience outcome of a reinforced Bioscaffold in inguinal hernia repair: a case series. Int J Surg Open 12:9-11 